\title{
HYBRID MONTE CARLO ALGORITHM AS APPLIED TO DYNAMICAL WILSON FERMION ${ }^{*}$ )
}

\author{
Akira Ukawa $\left.{ }^{+}\right)$
}

CERN - Geneva

\section{A B S T R A C T}

The hybrid Monte Carlo algorithm is tested for lattice QCD with Wilson quark action. A variety of technical points are examined. As an application the finite temperature behaviour of QCD with Wilson quark action is studied.

*) To appear in the Proceedings of the 1988 Symposium on Lattice Field Theory, FNAL, September 22-25, 1988.

+ ) On leave from Institute of Physics, University of Tsukuba, Ibaraki 305, Japan. 


\title{
HYBRID MONTE CARLO ALGORITHM AS APPLIED TO DYNAMICAL WILSON FERMION
}

\author{
Akira Ukawał \\ Theory Division, CERN, Geneva, Switzerland
}

\begin{abstract}
The hybrid Monte Carlo algorithm is tested for lattice QCD with Wilson quark action. A variety of technical points are examined. As an application the finite temperature behavior of QCD with Wilson quark action is studied.
\end{abstract}

\section{Motivation.}

The Langevin ${ }^{1}$ and hybrid ${ }^{2}$ algorithms have been quite successful in unravelling the characteristic features of the dynamical quark effect in lattice QCD. They suffer, however, from one drawback that the observable averages are contaminated by a systematic error for a finite step size $\Delta \tau$. The magnitude of this error can be roughly estimated as $\Delta \tau^{n} / \lambda_{\min }^{2}$ with $\lambda_{\min }$ the minimum eigenvalue of the lattice Dirac operator, and $n=1$ for Langevin and $n=2$ for hybrid algorithm. Since the minimum eigenvalue behaves as $\lambda_{\min } \propto m_{q} a$, the problem becomes quite severe as one tries to penetrate into the region of small quark mass. In the Langevin simulation this is already substantial ${ }^{3-5}$ at the quark mass of 0.1 for the standard step size $\Delta \tau=$ 0.01 , necessitating an extrapolation in $\Delta \tau$ to obtain a bias free result. The hybrid algorithm suffers less due to the error being second order. However, the relaxation is much slower than Langevin and an order of magnitude longer run is needed to achieve the same statistical accuracy ${ }^{6}$. Furthermore an extrapolation in $\Delta \tau$ will eventually become necessary even for the hybrid algorithm as the quark mass decreases below 0.01 .

The hybrid Monte Carlo algorithm(HMC $)^{7}$ offers an attractive alternative since it is in principle bias free for any step size. We have made a variety of tests of the algorithm for the Wilson quark action with two dynamical quark flavors, and the main points of the analysis are presented here. Part of the reason for working with the Wilson action comes from the fact that the systematic error problem is worse $e^{5}$ than the Kogut-Susskind case. This is due to the additional factor of $2 K$ in the relation $\lambda_{\min } \sim 2 K m_{q} a=1-K / K_{c}$ which is absent for the latter. As an application of HMC, we have studied the finite temperature behavior with the Wilson quark action. Some preliminary results are reported.

\section{Test of HMC.}

\subsection{Implementation.}

For the molecular dynamics evolution we used the simplest leap-frog procedure assigning the link variables to half-integer time and the conjugate momenta to integer time. We did not shift the coupling $\beta$ and the hopping parameter $K$ in the molecular dynamics steps. We use the notation $\left[\Delta \tau_{M C}, \Delta \tau_{m d}\right]$ for the step size parameters where $\Delta \tau_{m d}$ is for the molecular dynamics evolution, and $k=\Delta \tau_{M C} / \Delta \tau_{m d}$ is the number of steps between the refreshing of the momenta and the pseudofermion variable $Y$. The inversion of the quark matrix $Y \rightarrow x=D_{q}^{-1} Y$ was made by the ILUCR algorithm described in detail in ref. 3 . We denote the stopping condition by $\left(r_{M C}, r_{m d}\right)$ where $r=\left\|Y-D_{q} x\right\| / \sqrt{3 \cdot 4 \cdot V}$ with $V$ the lattice volume.

\subsection{Comparison with Langevin.}

We made HMC runs with $N_{f}=2$ flavors on an $8^{4}$ lattice at $K=0.14,0.15,0.16$ with $\beta=5.5$, using the periodic boundary condition in all four directions. We started from a thermalized pure gauge configuration,

$\dagger$ On leave from Institute of Physics, University of Tsukuba, Ibaraki 305, Japan. 
Table 1.

HMC run on an $8^{4}$ lattice with $N_{f}=2$ at $\beta=5.5$. The averages are taken over the last 300 trajectories out of 400. For Wilson loops, the HMC values are listed in the first row. The error is statistical only. The numbers in the second row are the Langevin result ${ }^{3}$ extrapolated to the zero step size. The critical hopping parameter is $K_{c}=0.1613(2)$.

\begin{tabular}{cllll}
$K$ & 0.14 & 0.15 & 0.16 \\
\hline step size & {$[1,0.05]$} & {$[1,0.05]$} & {$[0.5,0.025]$} \\
$r_{M C}=r_{\text {md }}$ & $4.5 \cdot 10^{-6}$ & $4.5 \cdot 10^{-6}$ & $4.5 \cdot 10^{-6}$ \\
acceptance & $56 \%$ & $45 \%$ & $74 \%$ \\
$<e^{-\delta H}>$ & $0.97(10)$ & $1.01(13)$ & $0.93(4)$ \\
$W_{1 \times 1}$ & HMC & $0.52350(16)$ & $0.53694(22)$ & $0.56018(18)$ \\
& L & $0.52367(80)$ & $0.53893(96)$ & $0.56454(77)$ \\
$W_{2 \times 2}$ & HMC & $0.10498(22)$ & $0.12141(30)$ & $0.15452(30)$ \\
& L & $0.10448(94)$ & $0.12279(121)$ & $0.15949(109)$ \\
$W_{3 \times 3}$ & HMC & $0.01079(13)$ & $0.01607(17)$ & $0.03089(19)$ \\
& L & $0.01044(50)$ & $0.01606(59)$ & $0.03169(71)$ \\
$W_{4 \times 4}$ & HMC & $0.00057(11)$ & $0.00129(12)$ & $0.00488(13)$ \\
& L & $0.00086(40)$ & $0.00137(47)$ & $0.00503(41)$
\end{tabular}

and chained in $K$ from $K=0.14$ to $K=0.16$ with 200 trajectories at each $K$. Another 200 trajectories were then added to check the thermalization. The simulation parameters together with the acceptance and the average of $e^{-\delta H}$ (which should be unity in equilibrium ${ }^{8,9}$ ) over the last 300 trajectories are listed in Table 1.

In Table 1 we also show the Wilson loops from the present $\mathrm{HMC}$ run and compare them with the Langevin result ${ }^{3}$ on a $9^{3} \times 18$ lattice obtained by a linear extrapolation from the range $\Delta \tau=0.02-0.005$. Overall we see a good agreement. This provides a non-trivial check of the exactness of HMC as well as the degree of control over the extrapolation in the Langevin run. The latter is rather remarkable in view of the long distance of extrapolation over which large Wilson loops increase by a factor of two or more. On a closer inspection, however, one may detect a slight difference at $K=0.16$ especially for small loops. This is partly due to a slow relaxation of the HMC run for $K=0.16$; the time history shows a systematic drift over the entire length of the run of 400 trajectories. This may not be surprising since, with ${ }^{4}$ $K_{c}=0.1613(2)$, the quark mass at $K=0.16$ is quite small; $m_{q} a=1 / 2\left(1 / K-1 / K_{c}\right)=0.025$.

At $K=0.15$ and 0.16 we also measured the hadron masses for the HMC run taking every 10th trajectory after the $200 \mathrm{th}$, and periodically extending the lattice from $8^{4}$ to $8^{3} \times 16$. For mesons, the result from a single pole fit over $t \geq t_{\min }=5$ (the same as in ref. 3 ) is $m_{\pi} \mathrm{a}=1.181(19)(1.142(28)), m_{\rho}=1.239(18)(1.190(32))$ at $K=0.15$, and $m_{\pi} \mathrm{a}=0.448(72)(0.348(30)), m_{\rho}=$ $0.661(42)(0.532(40))$ at $K=0.16$, where the error was estimated by dividing the sample into two bins. The numbers in the parentheses are the Langevin estimate ${ }^{3}$ extrapolated to the zero step size. We again see a nice agreement at $K=0.15$. At $K=0.16$, the hadron propagators in HMC markedly suffer from long range fluctuations. This is reflected in a large error, showing that the statistics is not enough. For this reason we regard the discrepancy as not yet serious. For baryons $N_{t}=16$ was too short to apply a single pole fit with $t_{\min }=6$ to compare with ref. 3 .

\subsection{Acceptance.}

We carried out a number of HMC runs with the volume ranging from $6^{4}$ to $8^{4}, \beta$ from 4.5 to 5.5 , and the step size in the interval $\Delta \tau_{m d}=0.0125-0.05$ and $\Delta \tau_{M C}=0.25-1.0$. The acceptance data collected shows the following noteworthy features; (i) The acceptance drops toward $K \rightarrow K_{\mathrm{c}}$ for fixed $\Delta \tau_{m d}$ (It is only weakly dependent on $\Delta \tau_{M C}$ since the Hamiltonian only oscillates around an average value for the leap-frog discretization ${ }^{2,10}$ ). (ii) However, with $\lambda_{\min }=1-K / K_{c}$ and the step size fixed, the acceptance increases with increasing $\beta$. This is probably due to the fact that the configuration becomes smoother and the change of Hamiltonian becomes less over a trajectory of fixed length. (iii) At $\beta=5.5$ on an $8^{4}$ lattice, an acceptance beyond $50 \%$ level is possible with $\Delta \tau_{m d} \sim 0.025$ even at the quark mass of $m_{q} a \sim 0.025\left(K=0.16, K_{c}=\right.$ $0.1613(2))$. Combined with an asymptotic estimate ${ }^{8,11}$ $P_{a c c} \sim e^{-c \Delta \tau_{m d}{ }^{4} V}$, a moderate step size of $\Delta r_{m d} \sim 0.01$ 
may be sufficient to maintain a reasonable acceptance on a lattice as large as $16^{4}$.

\subsection{Relaxation rate.}

HMC and the hybrid algorithm differs only by the global accept/reject step. We therefore expect the relaxation of HMC to be slowed down by the amount of acceptance compared to hybrid. Since the hybrid is slower than the Langevin ${ }^{6}$, the HMC has even longer range of fluctuations compared to the latter. These features were seen in our test runs.

An important question is the critical slowing down toward $K \rightarrow K_{c}$. Measured in units of $\tau_{H M C}=$ number of trajectories $\times \Delta \tau_{M C} \times$ acceptance, the two HMC runs at $K=0.15$ and 0.16 described in 2.2 above have similar length $\approx 150$. The fluctuation period in this unit is at least twice longer for $K=0.16$. Since $\Delta \tau_{m d}$ was halved to save the acceptance keeping $\Delta \tau_{M C} / \Delta \tau_{m d}$ fixed, one probably has to extend the number of trajectories $4-5$ times to obtain a reasonable statistical accuracy at $K=$ 0.16 . This exemplifies that the number of trajectories needed grows quite fast toward small quark masses due to the combined effect of critical slowing down and the loss of acceptance.

\subsection{Precision of matrix inversion.}

For the global accept/reject step, one needs to calculate $D_{q}^{-1} Y$ quite accurately so that the change of the Hamiltonian $\delta H$ for each trajectory is correct within the width of its distribution over the ensemble of trajectories. We estimate that our stopping condition $r_{M C}<$ $4.5 \cdot 10^{-6}$ on $8^{4}$ corresponds to a relative accuracy of $<5 \%$ for $\delta H$. Much more subtle is the error for the molecular dynamics step. Contrary to a naive suspicion, this may not introduce an uncontrollable bias if an approximate inversion actually corresponds to solving for some other matrix which, however, depends only on the current gauge configuration ${ }^{8}$. A practical check for this point is provided ${ }^{8}$ by the identity ${ }^{8}\left\langle e^{-\delta H}\right\rangle=1$. Our runs are consistent with this identity, though with a large error, except possibly at $K=0.16$ (see Table 1).

\subsection{Computing cost.}

The number of matrix inversions for each update which dominate the computer time is the same for the
Langevin (partial second order scheme ${ }^{3}$ ), hybrid and HMC. One feature which possibly makes HMC more time consuming is the necessity of a more precise matrix inversion. Against Langevin (with $\Delta r$ extrapolation to control the systematic error), there is an additional factor of a slower relaxation. To obtain an idea on these points, we quote the relevant numbers for the Langevin ${ }^{3,4}$ and HMC simulations of Table 1 at $K=0.16$; For the former, the total number of sweeps summing over the three values of $\Delta \tau$ used is 17,500 as compared to 8,000 for HMC. The average number of steps for the matrix inversion was $25-35$ for Langevin and 80-90 for HMC. The increase partly comes from a more stringent stopping condition for HMC $\left(r<4.5 \cdot 10^{-6}\right.$ as compared to $\left.<2.5 \cdot 10^{-3}\right)$ and is partly due to the underestimate of the quark loop effect in the Langevin simulation for finite $\Delta \tau$. If one has to extend the HMC run 4-5 times to bring the statistical fluctuation under control, as we indicated above, then HMC is roughly six times more costly than the Langevin simulation. Comparison with the hybrid algorithm (without extrapolation) is more straightforward since it only depends on the stopping condition and the acceptance. With the value $74 \%$ for the latter, we estimate a factor 4 increase for $\mathrm{HMC}$ if we take over the stopping condition of the Langevin run for the hybrid.

\subsection{Summary.}

HMC works reasonably well on a lattice such as $8^{4}$ for the Wilson quark action. It does require more computer time than the approximate algorithms, however. The basic reasons are that (i) the problem of critical slowing down toward $K=K_{c}$ is enhanced by the need to reduce the step size to maintain acceptance, and (ii) the matrix inversion has to be carried out more precisely. Both these problems will become more severe as the lattice size is increased. This then will be the cost of working with a bias-free algorithm at small quark masses. In this respect, a careful scrutiny of how much one can relax the precision of the matrix inversion in HMC will be worthwhile since it directly controls the computer time. 
3. Finite temperature behavior with Wilson action.

Finite temperature simulations with the KogutSusskind action have shown that a first order chiral transition of an increasing strength hits $m_{q} a=0$ at a finite value of $\beta$. A naive identification of the vanishing quark mass $m_{q} a=0$ with the critical hopping parameter $K=K_{c}$ of the Wilson action will lead one to expect that a transition of an increasing chiral character crosses the line $K=K_{c}(\beta)$ in the Wilson case. A Langevin simulation carried out some time ago ${ }^{12}$ on a small lattice of $5^{3} \times 3$ with $N_{f}=4$ flavors has indicated that this might not be the case; it was found that the transition line did not appear to cross $K=K_{c}$, but rather ran parallel to it reaching down the strongcoupling limit $\beta=0$, where no sign of a first order transition was seen. We have attempted a similar analysis with HMC on a slightly larger lattice $8^{3} \times 4$ with two dynamical flavors. For recent similar attempts, see refs. 13-14.

\subsection{Data taking.}

The HMC parameters are $[0.5,0.025]-[0.25,0.025]$ and $r_{m d}=r_{M C}=6.4 \times 10^{-6}$. We worked at $\beta=$ 5.5, 5.0 and 4.5 starting from a pure gauge configuration and increasing the hopping parameter. The number of trajectories at each $K$ is generally $400-1,000$ with the acceptance of $50-90 \%$, except at $\beta=4.5$ and $K \geq 0.2$ where the acceptance became so low $(<30 \%)$ that we were forced to switch to the hybrid algorithm with some risk of systematic error. We have also made a separate hadron mass measurement on a $6^{3} \times 12$ lattice at $\beta=5.0$ and 4.5 to estimate $K_{c}$. At $\beta=5.5$ we used the Langevin value ${ }^{4}$. See Table 2.

\subsection{Phase diagram.}

We show in fig. 1 the phase diagram for $N_{f}=2$ Wilson quark action at the temporal lattice size $N_{t}=4$. The squares denote the measured points of the critical hopping parameter and the solid line is drawn to guide the eye. The horizontal bars show the interval of $K^{n}$ over which the Polyakov line (normalized to unity) made a rapid rise from a small $(<0.05)$ to large $(>0.1)$ value. Other observables including $\langle\bar{\psi} \psi\rangle$ and the energy densities changed simultaneously. At $\beta=5.5$ we made flip-flop searches in steps of $\delta K=0.025$ be-

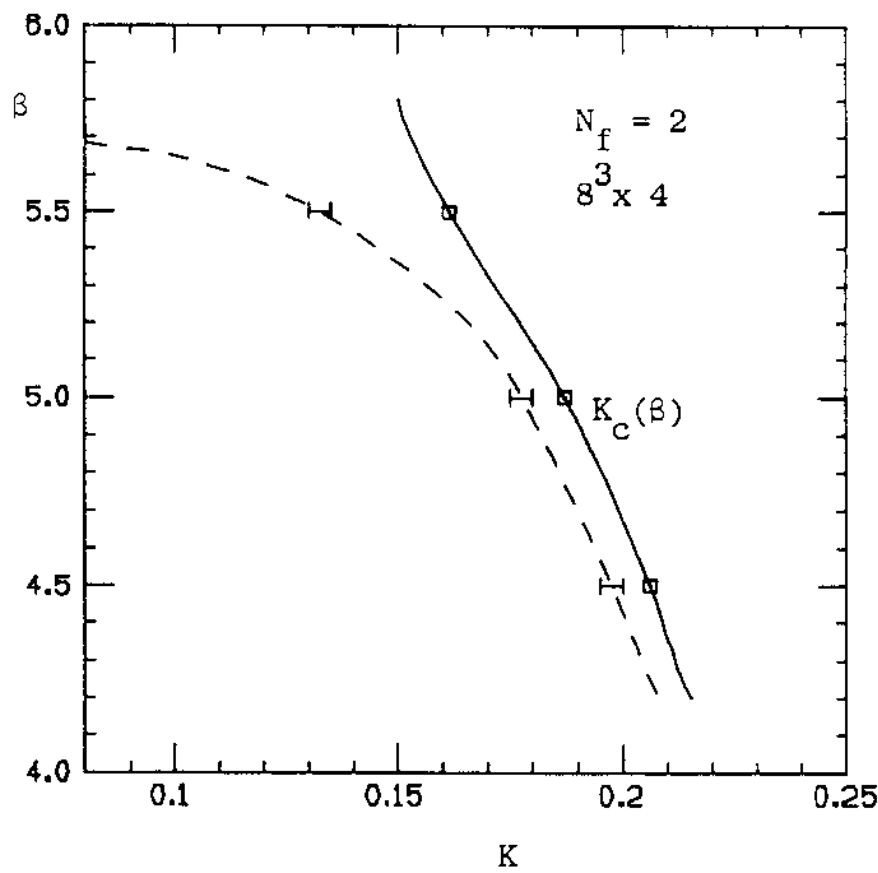

Fig. 1

Finite temperature phase diagram for Wilson fermion with $N_{f}=2$ obtained on an $8^{3} \times 4$ lattice. The squares with a solid line interpolation are the critical hopping parameter. The horizontal bars are the location of the transition. The dashed line is an interpolation.

Table 2.

Parameters relevant for the finite temperature transition with Wilson quark action for $N_{f}=2 . m_{\rho}=$ $0.77 \mathrm{GeV}$ is used to estimate the lattice spacing. $K_{t r}$ denotes the estimated position of transition. We use $m_{q}=1 / 2 a\left(1 / K-1 / K_{c}\right)$ for the quark mass. The values of $m_{q}$ and $m_{\pi}$ correspond to the middle of the transition region.

\begin{tabular}{llll}
\hline$\beta$ & 5.5 & 5.0 & 4.5 \\
\hline$K_{c}$ & $0.1613(2)$ & $0.187(1)$ & $0.206(2)$ \\
$a^{-1}(\mathrm{GeV})$ & $1.75(13)$ & $0.99(6)$ & $0.73(6)$ \\
$K_{t r}$ & $0.130-0.135$ & $0.175-0.180$ & $0.195-0.200$ \\
$m_{q}(\mathrm{GeV})$ & 1.18 & 0.14 & 0.076 \\
$m_{\pi}(\mathrm{GeV})$ & 3.36 & 0.94 & 0.67 \\
\hline
\end{tabular}


tween $K=0.13$ and 0.14 with $600-1,000$ trajectories and found some indication at $K=0.1300-0.1350$. An apparently wide critical region is probably due to the fact that the line of constant $\beta$ followed in the search cuts the transition line at a small angle (see fig. 1 ). At $\beta=5.0$, we find a signature of flip-flop at $K=0.1775$, while at $\beta=4.5$ we have not seen any up to $K=0.20$ within our limited statistics.

Fig. 1 shows that the transition does approach the line of critical hopping parameter. To put this on a more quantitative basis we quote the quark mass $m_{q}=1 / 2 a\left(1 / K-1 / K_{c}\right)$ and the pion mass at zero temperature at the transition point in Table 2 where the lattice spacing is determined by $m_{\rho}=0.77 \mathrm{GeV}$ at $K=K_{c}$. These mass values are fairly large even at $\beta=4.5$, which by themselves may be sufficient to exclude a clear chiral transition. In addition, the transition does not show any apparent tendency to cross the line $K=K_{\mathrm{c}}$. The behavior on the $N_{t}=4$ lattice with $N_{f}=2$ therefore remains qualitatively the same as on the $N_{t}=3$ lattice with $N_{f}=4$ studied in ref. 12 .

Let us now speculate on a possibility that this troublesome behavior might change for a larger $N_{t}$. We start by recalling that the lattice is an ultra-violet regulator. With the Wilson quark action this is a chirally non-invariant one. To extract the true chiral content, one therefore needs to separate out the cut-off dependent regularization effects which do not respect chiral symmetry. A nice method is provided by the Ward identity analysis ${ }^{15}$. This analysis shows that the chiral symmetry breaking along $K=K_{c}$, which remains after an appropriate (generally non-perturbative) renormalization of the currents and pseudoscalar densities, is an $O(a)$ correction, and therefore decreases toward the continuum limit. Away from $K=K_{c}$ there will be an additional breaking due to a non-vanishing quark mass.

Consider now the transition line $\beta=\beta_{c}(K)$ on the $(\beta, K)$ plane. For $N_{t}=4$ this is the dashed line interpolating the bars in fig. 1 and reaching the pure gauge deconfinement point at $K=0$. If we increases $N_{t}$, the transition line will move up toward larger $\beta$. This means in particular that its shoulder where it approaches $K=K_{c}$ will take place at a larger value of $\beta$, and probably closer to $K=K_{c}$. The regularizationdependent chiral breaking will therefore be less in magnitude. For large enough $N_{t}$ the corresponding value of $\beta$ for the shoulder will be in the scaling region. The magnitude of breaking $O(a) \sim e^{-1 / 2 b_{0} g^{2}}$ may then be small enough to allow a chiral transition to take place for the Wilson action as well. Once this occurs, the transition line will cross $K=K_{c}(\beta)$ since the total chiral breaking (quark mass and $O(a)$ regularization effect) is smallest along it.

One may try to estimate how large the temporal size has to be for this to happen. One way is to examine how much the transition line of fig. 1 should be shifted as a whole toward larger $\beta$ before it intersects the critical line $K=K_{c}$. This requires $\delta \beta \approx+0.15-0.2$. For the pure gauge deconfinement point at $K=0$, an increase of $\beta_{\mathrm{c}}$ by this amount occurs for $N_{t}=5-6$. This is an underestimate since the amount of shift for the actual transition line will decrease for larger $K$. Another way $^{16}$ is to use the relation $T_{c}=1 / a\left(\beta_{c}\right) N_{t}$. From the Kogut-Susskind simulation with two flavors ${ }^{17}$, we have an estimate $T_{c} \approx 150 \mathrm{MeV}$. On the other hand, a Langevin simulation with Wilson quark action with the same number of flavors ${ }^{4}$ gave $1 / a(\beta=5.5) \approx 1.75 \mathrm{GeV}$. For the transition line to cross $K=K_{c}$ at $\beta=5.5$ one then needs $N_{t}=11-12$. This estimate is much less certain for several reasons; it assumes $T_{c}=150 \mathrm{MeV}$ to be common between the Kogut-Susskind action at $\beta=5.25-5.4$ where the estimate was made and the Wilson action at $\beta=5.5$. This range of $\beta$ is too low for the scaling to have set in, allowing a discrepancy of perhaps $20-30 \%$. It also assumes that the chiral breaking in the Wilson action is already small at $\beta=5.5$. This may not be altogether wrong as we shall discuss below, however. Allowing for these margins of uncertainty, one needs at least $N_{t}=6$ and more likely $N_{t}=8-10$ to see whether the chiral transition actually takes place with the dynamical Wilson fermions.

3.3. Hadronic screening length.

An interesting observable recently calculated in the Kogut-Susskind finite temperature simulation is the screening length for a variety of hadronic charges ${ }^{18}$. We have repeated the calculation with the Wilson quark ac- 
tion at $\beta=5.5$. For this purpose we took every 10 th configuration from our run, and measured the spatial hadron propagators on an $8^{2} \times 16 \times 4$ lattice constructed by periodically doubling the original $8^{3} \times 4$. In fig. 2 the results for mesonic channels are shown in the form of mass, and are compared with the zero temperature ones obtained on a $9^{3} \times 18$ lattice ${ }^{3}$. After $K$ crosses the finite temperature transition at $K_{t r}=0.130-0.135$, the pion mass ceases to decrease and clearly stays finite at $K=K_{c}$. More interestingly, the parity partners $\pi-\sigma$ and $\rho-A_{1}$, which are separate for the zero temperature case, show a clear tendency of degeneracy toward $K=K_{\mathrm{c}}$. This feature was also seen for the baryons $N_{+}-N_{-}$and $\Delta_{+}-\Delta_{-}$. This is the same characteristic as was found for the Kogut-Susskind case. It indicates that the chiral symmetry is restored in the high temperature phase and as a prerequisite the $O(a)$ chiral symmetry breaking may already be fairly small at $\beta=5.5$ with $1 / a \approx 1.75 \mathrm{GeV}$.

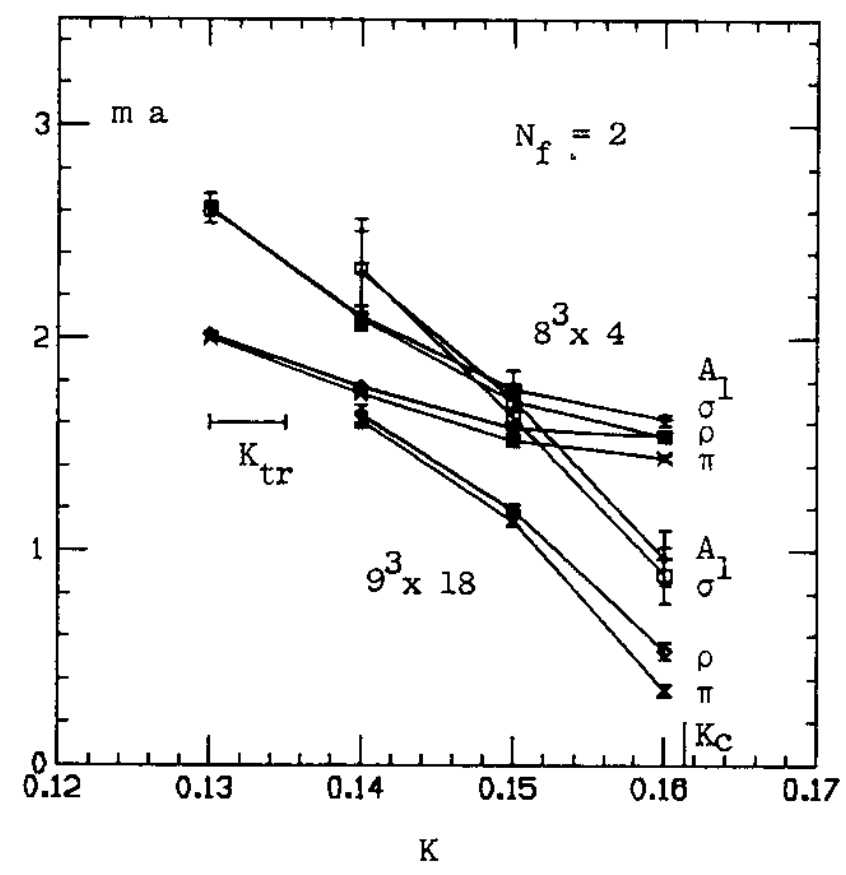

Fig. 2

Comparison of meson masses at zero $\left(9^{3} \times 18\right)$ and finite $\left(8^{3} \times 4\right)$ temperatures as a function of the hopping parameter. The horizontal bar on the left indicates the position of transition. The lines are drawn to guide the eye.
Acknowledgements.

We thank R. Gupta, Y. Iwasaki, F. Karsch and A. Patel for informative discussions. We are grateful to the Theory Division of CERN where the present work was carried out for its hospitality. Numerical calculations were made on the CRAY-XMP48 installed at CERN early this year.

References.

1. A. Ukawa and M. Fukugita, Phys. Rev. Lett. 55 (1985) 1854; G. G. Batrouni et al., Phys. Rev. D32 (1985) 2736.

2. S. Duane, Nucl. Phys. B257[FS14] (1985) 652; S. Duane and J. B. Kogut, Nucl. Phys. B275[FS17] (1986) 398;

S. A. Gottlieb et al., Phys. Rev. D35 (1987) 2531.

3. M. Fukugita, Y. Oyanagi and A. Ukawa, Phys. Rev. Lett. 57 (1986) 953; Phys. Rev. D36 (1987) 824.

4. M. Fukugita, Y. Oyanagi and A. Ukawa, Phys. Lett. 203B (1988) 145.

5. M. Fukugita and A. Ukawa, preprint RIFP-734 (1988), Phys. Rev. D, in press.

6. M. Fukugita and A. Ukawa, Nucl. Phys. B300 [FS29] (1988) 433.

7. S. Duane et al., Phys. Lett. 195B (1987) 216.

8. M. Creutz, Phys. Rev. D38 (1988) 1228.

9. R. Gupta and A. Patel, private communication.

10. M. Fukugita, T. Kaneko and A. Ukawa, Nucl. Phys. B270[FS16] (1986) 365.

11. R. Gupta et al., Phys. Rev. D38 (1988) 1278.

12. M. Fukugita, S. Ohta and A. Ukawa, Phys. Rev. Lett. 57 (1986) 1974.

13. Y. Iwasaki and X. Tsuboi, preprint UTHEP-185 (1988).

14. A. Patel et al, in these Proceedings and preprint LA-UR-88-998 (1988).

15. M. Bochicchio et al., Nucl. Phys. B262 (1985) 331.

16. Y. Iwasaki, private communication.

17. S. A. Gottlieb et al., Phys. Rev. Lett. 59 (1987) 1513; preprint FERMILAB-PUB-88/62/T (1988).

18. C. DeTar and J. B. Kogut, Phys. Rev. Lett. 59 (1987) 399; Phys. Rev. D36 (1987) 2828;

S. A. Gottlieb et al., Phys. Rev. Lett. 59 (1987) 1881. 